\title{
In Vivo Osteogenesis of Vancomycin Loaded Nanohydroxyapatite/Collagen/Calcium Sulfate Composite for Treating Infectious Bone Defect Induced by Chronic Osteomyelitis
}

\author{
Xiaojie Lian, ${ }^{1}$ Kezheng Mao, ${ }^{2}$ Xi Liu, ${ }^{3,4}$ Xiumei Wang, ${ }^{4}$ and Fuzhai Cui ${ }^{4}$ \\ ${ }^{1}$ College of Mechanics, Taiyuan University of Technology, Taiyuan 030024, China \\ ${ }^{2}$ Zhengzhou Orthopedics Hospital, Zhengzhou 450052, China \\ ${ }^{3}$ National Engineering Laboratory for Modern Silk, College of Textile and Clothing Engineering, Soochow University, \\ Suzhou 215123, China \\ ${ }^{4}$ State Key Laboratory of New Ceramics and Fine Processing, Department of Materials Science and Engineering, \\ Tsinghua University, Beijing 100084, China
}

Correspondence should be addressed to Xiaojie Lian; yuhalian@126.com

Received 31 August 2014; Accepted 24 October 2014

Academic Editor: Xiaoming Li

Copyright (C) 2015 Xiaojie Lian et al. This is an open access article distributed under the Creative Commons Attribution License, which permits unrestricted use, distribution, and reproduction in any medium, provided the original work is properly cited.

A novel antibacterial bone graft substitute was developed to repair bone defects and to inhibit related infections simultaneously. This bone composite was prepared by introducing vancomycin (VCM) to nanohydroxyapatite/collagen/calcium sulphate hemihydrate (nHAC/CSH). XRD, SEM, and CCK-8 tests were used to characterize the structure and morphology and to investigate the adhesion and proliferation of murine osteoblastic MC3T3-E1 cell on VCM/nHAC/CSH composite. The effectiveness in restoring infectious bone defects was evaluated in vivo using a rabbit model of chronic osteomyelitis. Our in vivo results implied that the $\mathrm{VCM} / \mathrm{nHAC} / \mathrm{CSH}$ composite performed well both in antibacterial ability and in bone regeneration. This novel bone graft substitute should be very promising for the treatment of bone defect-related infection in orthopedic surgeries.

\section{Introduction}

Bone defect-related infections especially chronic osteomyelitis are quite common in open fracture and trauma in clinical treatment, which continues to be very difficult to treat and brings challenges to clinicians. It is difficult to make effective bone repair and inhibit infection at the same time. Vancomycin hydrochloride (VCM) is an antibiotic drug that is specifically used against Staphylococcus aureus in the treatment of bone-related infections. However, systemically administered antibiotics have been associated with a number of difficulties including toxic side effects if the drug level is too high or may fail to exert the proper therapeutic effect if the drug level is too low at the site of need. These disadvantages could be markedly reduced if the antibiotic is applied locally at the site of infection by incorporating it into, or onto, implantable skeletal delivery scaffold, and it may also improve efficacy by delivering valid and safe drug concentrations to the infected bone.

Therefore, it is critical to select an appropriate scaffold for constructing drug delivery system, in which the scaffold should be biocompatible, osteogenic, operable, biodegradable, and antibacterial. Primarily, the scaffold should possess suitable ingredients and structure for cell attachment, proliferation, and osteogenic differentiation. Li et al. did some related studies [1-3].

PMMA is often incorporated with antibiotics such as gentamicin as one of the most widely used bone materials in clinical applications. However, the inherent biological inertia of PMMA leads to poor osseointegration between bone tissue and cement interface, apart from other shortcomings such as nonabsorbability, impermeability to antibiotic, monomer 
toxicity, and high polymerization temperature [4-8]. Because of these drawbacks, several absorbable, osteoconductive, and low reaction temperature antibiotic carriers have been developed for inhibiting infection as well as avoiding a second surgery [9-14].

Calcium sulfate hemihydrate (CSH) itself has a long clinical history as a bone graft substitute, known for its bioresorption, satisfactory handling properties, or self-setting ability in situ after filling the defect. After mixing, CSH form a viscous moldable paste, which in some instances can be injected during surgery using minimally invasive procedures. Moreover, the setting reaction of $\mathrm{CSH}$ is not obviously exothermic as PMMA, which are widely used in orthopedic surgery, especially for arthroplasty fixation and vertebroplasty. Therefore, the incorporation of different drugs and biological molecules makes them good candidates for drug delivery applications in bone tissue engineering [15]. However, there are also some drawbacks with the calcium sulfate material including its insufficient ability to stimulate bone regeneration. $\mathrm{CSH}$ cement cannot form a chemical bond with bone tissue at the early stage of therapy because of its poor bioactivity [16-19]. Ideal antibiotic carriers should be able to promote early mineralization and support new bone formation and simultaneously control the release of drug.

To optimize the performance of bone regeneration of $\mathrm{CSH}$, some previous studies in our group suggested that nanohydroxyapatite/collagen (nHAC) could be incorporated, which was prepared from mineralizing type I collagen with excellent osteoconductive properties, and was thought to be a new scaffold material for its high similarity of natural bone both in composition and in hierarchical nanostructure in bone tissue engineering [20-25]. It has been demonstrated that nanostructured materials, compared with conventional materials, may promote greater amounts of specific protein interactions, thereby more efficiently stimulating new bone formation. It has also been indicated that when features or ingredients of scaffolds are nanoscale, a variety of interactions can be stimulated at the cellular level [26-29].

The aim of the present study was to investigate nHAC/ $\mathrm{CSH}$ as a carrier material for vancomycin in the treatment of chronic osteomyelitis to repair bone defects and inhibit related infections simultaneously. An in vivo study of the efficacy of this drug-loaded bone cement was performed in an experimental model of chronic bone infection caused by MRSA.

\section{Materials and Methods}

2.1. Material Preparation and Physicochemical Properties Characterization. The powders of $\mathrm{nHAC}$ and $\mathrm{CSH}$ were prepared as previously described. In brief, nHAC were obtained by precipitation of $\mathrm{Ca}^{2+}$ and $\mathrm{PO}_{4}{ }^{3+}$ on the collagen molecule from its precursors $\left(\mathrm{CaCl}_{2}\right.$ and $\left.\mathrm{NaH}_{2} \mathrm{PO}_{4}\right)$, followed by product freeze-drying. And the $\mathrm{CSH}$ were prepared from CSD (calcium sulphate dehydrate) by hydrothermal synthesis $[30,31]$. In this study, the VCM/nHAC/CSH were prepared by mixing $5 \mathrm{wt} \% \mathrm{VCM} \cdot \mathrm{HCl}, 5 \mathrm{wt} \% \mathrm{nHAC}$, and $90 \mathrm{wt} \% \mathrm{CSH}$ uniformly. Control samples (nHAC/CSH) were also prepared using the same process without VCM. The samples were mixed with deionized water at 0.5 ratio of liquid to solid (L/S) and stirred to form homogeneous paste within $20 \mathrm{~s}$ and then stored in $100 \%$ humidity at $37^{\circ} \mathrm{C}$ to set.

The composition of materials was characterized by $\mathrm{X}$ ray diffraction (XRD, D/max-2500X) using monochromated CuK $\alpha$ radiation $(\lambda=1.5405 \AA, 120 \mathrm{~mA}, 40 \mathrm{kV})$ in a continuous scan mode with a scanning speed of $8^{\circ} / \mathrm{min}$, and the $2 \theta$ range was from $10^{\circ}$ to $90^{\circ}$.

Composite samples were sputter-coated with gold film for scanning electron microscopy (SEM, Quanta 200 FEG, Netherlands) examinations at the voltage of $20 \mathrm{kV}$.

2.2. Cell Experiment. In order to evaluate the biocompatibility of cement, cell counting kit-8 (CCK-8) method was used to quantitatively evaluate the proliferation of murine osteoblastic MC3T3-E1 (a clonal osteogenic cell line derived from newborn mouse calvarias, which is often used in bone tissue engineering research). Cell growth and adhesion behaviors on the scaffolds surfaces were examined by SEM observation. MC3T3-E1 cells were cultured in Dulbecco's Modified Eagle Medium supplemented with 10\% fetal bovine serum, $50 \mu \mathrm{g} / \mathrm{mL}$ penicillin, and $50 \mu \mathrm{g} / \mathrm{mL}$ streptomycin at $37^{\circ} \mathrm{C}$ in a humidified atmosphere of $5 \% \mathrm{CO}_{2}$ and $95 \%$ air incubator. Two types of composite cements ( $\mathrm{nHAC/CSH}$ and $\mathrm{VCM} / \mathrm{nHAC} / \mathrm{CSH}$ ) were cut into small cubes (with $5 \mathrm{~mm}$ in diameter and $2 \mathrm{~mm}$ in thickness) for 96-well plate, sterilized by ${ }^{60} \mathrm{Co} \gamma$-irradiation at the dose of $16 \mathrm{kGy}$. The cells were seeded onto the sterilized cements at a density of $1 \times 10^{5}$ cells $/ \mathrm{mL}$, and $100 \mu \mathrm{L}$ of such cell suspension was added to each well. For SEM examination (JSM-T300, JEOL, Tokyo, Japan), cells were collected after $24 \mathrm{~h}$ incubation and then fixed with $2.5 \%$ glutaraldehyde in PBS for $0.5 \mathrm{~h}$. After dehydration with a series of graded ethanol $(30,50,70$, $80,90,95$, and $100 \%$ ), the samples were critical-point-dried and then coated with gold film for SEM examination. In order to evaluate cell proliferation, CCK-8 assay (Dojindo Molecular Technologies Inc.) was performed according to the manufacturer's protocol. Briefly, after incubating at $37^{\circ} \mathrm{C}$ and $5 \% \mathrm{CO}_{2}$ for indicated time $(1,3$, and 5 days), the culture medium was replaced with $100 \mu \mathrm{L}$ fresh medium containing $10 \mu \mathrm{L}$ CCK-8 solution in each well for another $3 \mathrm{~h}$ incubation before measurement at $450 \mathrm{~nm}$ with a micro plate reader (Bio-Rad, Model 680). Four repeated measurements for each time point of each group $(n=4)$ were carried out for statistical analysis.

2.3. In Vivo Study. The animal experiments were carried on by the approval of the Ethics Committee of the General Hospital of People's Liberation Army. The in vivo study was conducted on New Zealand rabbits $(3.5-4.0 \mathrm{~kg})$. Bone infection was induced in the condylus lateralis femoris in 30 rabbits using the model of Norden et al. under general anaesthesia. The site of operation was shaved and cleaned with alcohol. The medullary cavity was exposed through a lateral approach and a small hole was made $(\Phi 7 \mathrm{~mm} \times$ $9 \mathrm{~mm}$ ). $0.2 \mathrm{~mL} 5 \%$ sodium morrhuate was directly delivered into the cavity followed by $0.2 \mathrm{~mL}$ bacterial suspension with 


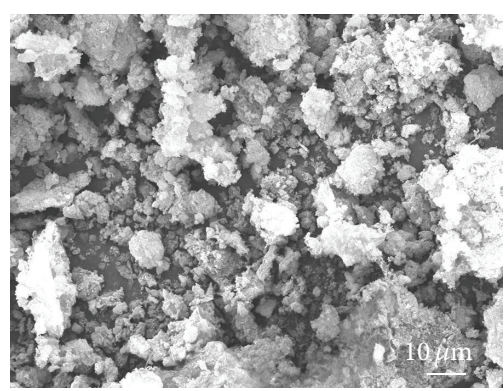

(a)

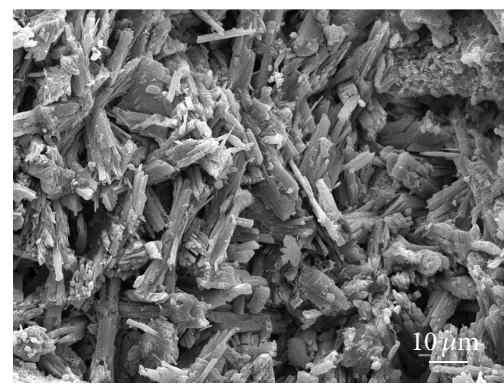

(b)

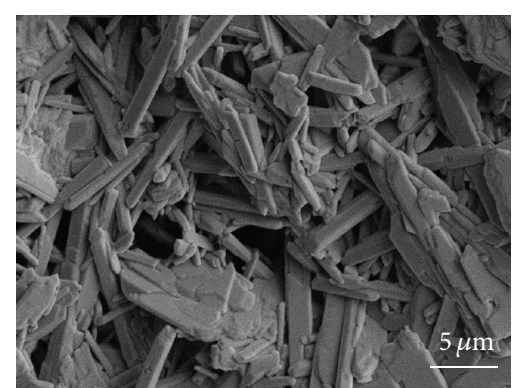

(c)

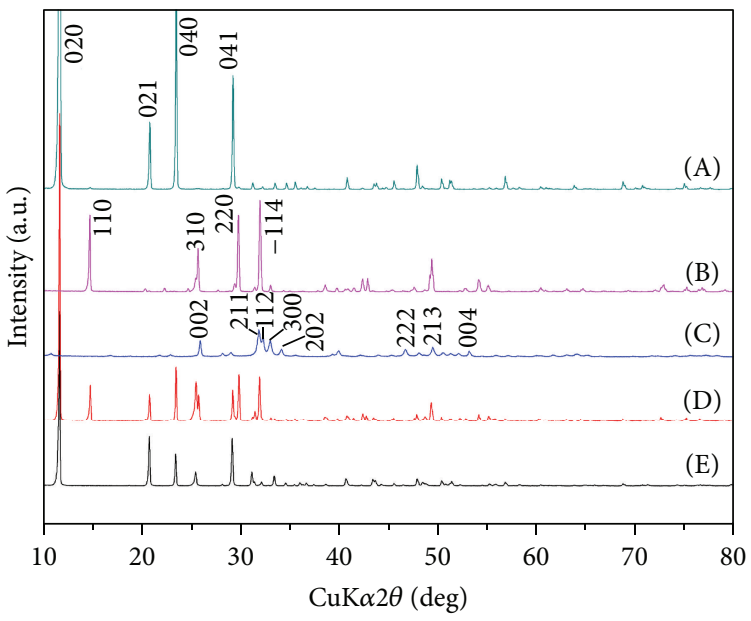

(d)

Figure 1: SEM micrographs of (a) nHAC, (b) CSH crystal, and (c) CSD crystal and XRD patterns of (d)(A) CSD, (d)(B) CSH, (d)(C) nHAC, (d)(D) compound of VCM/nHAC/CSH, and (d)(E) set cement of VCM/nHAC/CSH.

$1 \times 10^{8} \mathrm{CFU} / \mathrm{mL}$ S. aureus (MRSA, ATCC 43300, Shanghai Harmony Biotechnology Co., Ltd.). The wound was closed by suturing. Three weeks after induction of infection, all rabbits were infected and treated by focal debridement. Control group were injected by nHAC/CSH loading without antibiotics, whereas animals of treatment group were injected by $\mathrm{VCM} / \mathrm{nHAC} / \mathrm{CSH}$ for 3 months.

After surgery, rabbit femoris in defect regions were extracted and fixed in $4 \%$ paraformaldehyde and then decalcified in EDTA and dehydrated in ethanol before they were embedded in paraffin. Sections were prepared and stained with hematoxylin and eosin and Masson's trichrome. Histomorphometry was carried out using a light microscope (BX51, Olympus) under 40x magnification. For the evaluation of fibrosis, picrosirius red staining was performed using $0.1 \%$ picrosirius red solution. Micro-computed tomography was used to observe the bone reconstitution.

2.4. Statistical Analysis. All the data were statistically analyzed using SPSS 13.0 software and expressed as the standard deviation of the mean. The $t$-test was performed and $p<0.05$ was commonly accepted to be statistically significant.

\section{Result and Discussion}

3.1. Characterization of the Physicochemical Properties of $V C M / n H A C / C S H$ Scaffold. The conversion of CSD phase to $\alpha$-CSH phase by hydrothermal synthesis was confirmed by XRD analyses (Figures $1(\mathrm{~d})(\mathrm{A})$ and $1(\mathrm{~d})(\mathrm{B})$ ) and revealed complete transformation of CSD with new diffraction peaks located at $14.75^{\circ}, 25.58^{\circ}, 29.76^{\circ}$, and $31.94^{\circ}$, which were correlated with the characteristic crystal planes of 110, 310, 220 , and -114 for $\alpha$-CSH phase.

Also the characteristic peaks of nHAC located at $25.91^{\circ}$, $31.82^{\circ}, 32.20^{\circ}, 32.93^{\circ}$, and $34.10^{\circ}$ and correlated with crystal planes of 002, 211, 112, 300, and 202 (Figure $1(\mathrm{~d})(\mathrm{C})$ ) were shown. The broadening of the diffraction peaks of nHAC implied the small grain size and low crystallinity. nHAC powder was composed of some irregular particles as shown in Figure 1(b). The similarities to natural bone in the microstructure of nHAC have been verified via conventional and high-resolution transmission electron microscopy by Zhang et al. [32]. After the hydration reaction from $\mathrm{VCM} / \mathrm{nHAC/CSH}$ powder to set cement, the XRD peaks of which were changed to $11.64^{\circ}, 20.75^{\circ}, 23.41^{\circ}$, and $29.14^{\circ}$, corresponding to crystal planes of $020,021,040$, and 041 . Such changes indicated that the CSH with rod-like structure 


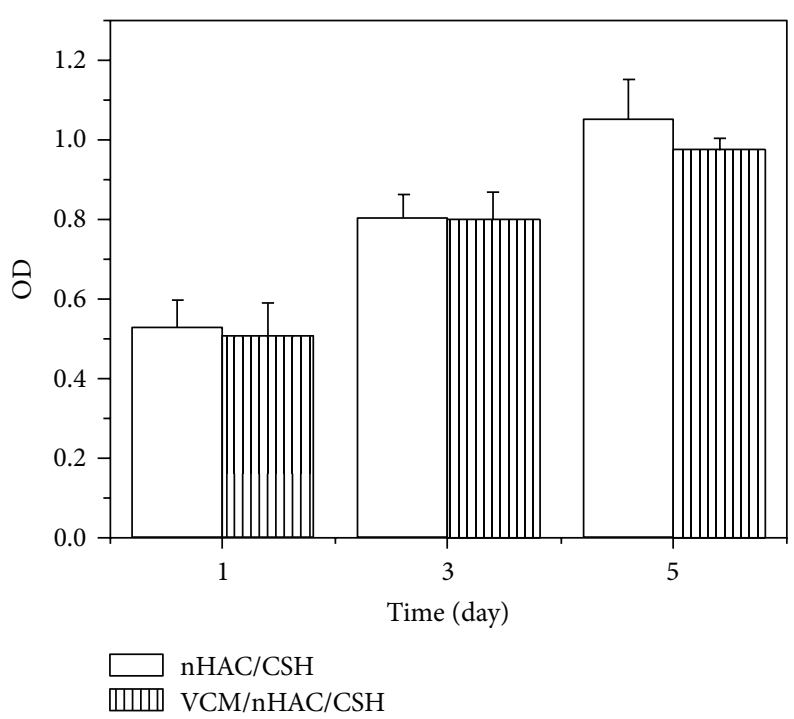

(a)

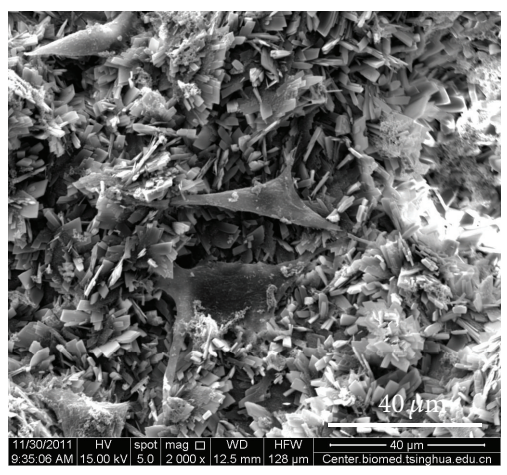

(b)

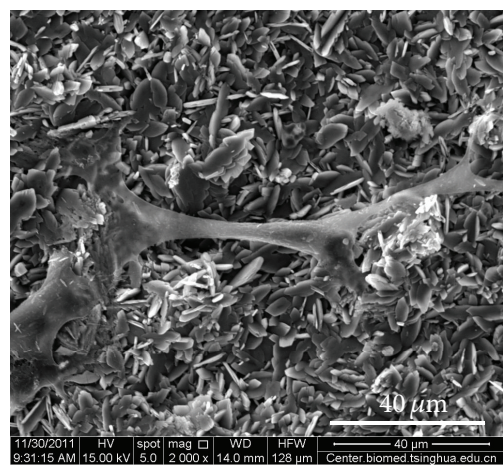

(c)

FIGURE 2: (a) Cells proliferation on the nHAC/CSH and VCM/nHAC/CSH scaffolds evaluated by CCK-8 assay and SEM micrographs of cells on (b) VCM/nHAC/CSH and (c) nHAC/CSH.

in the powder was transformed into CSD with sheet crystal structure in the set cement during the hydration reaction, as shown in Figures 1(b) and 1(c).

In our previous study, it was demonstrated that final setting time was about 15 20 min. The porosity of the scaffold was $38.8 \%$ and the compressive mechanical strength was about $4.8 \mathrm{MPa}$, which was more than the lower limit of natural cancellous bone $(1 \mathrm{MPa})$ [31].

3.2. CCK-8 Assay. In order to evaluate the cell toxicity of VCM, CCK-8 method was used to measure the proliferation of MC3T3-E1 cells on the scaffold surfaces. Figure 2(a) shows the proliferation results of cells cultured for 1,3 , and 5 days, respectively. For all times, the OD values of $\mathrm{VCM} / \mathrm{nHAC} / \mathrm{CSH}$ were not significantly lower than that of the $\mathrm{nHAC/CSH}$ for each point in time. This is not surprising since the antibacterial property of VCM is also expected to have an adverse effect on the osteoblastic cell viability. However, it was also shown that the cell growth on both groups increased significantly from 1 day to 5 days $(p<$ 0.05). Beyond that, cells performed well in attachment and spreading on both of bone materials with multiple filopodia. These results indicated that the VCM/nHAC/CSH scaffold with $5 \mathrm{wt} \%$ VCM addition had satisfied in vitro biocompatibility.

3.3. In Vivo Study. In the previous study, the materials were shown to be in vitro antibacterial. The inhibition ratio of $\mathrm{VCM} / \mathrm{nHAC} / \mathrm{CSH}$ was more than $99.8 \%$ and the distinct inhibition zone of $18 \mathrm{~mm}$ was formed in Staphylococcus aureus bacterium incubation dish with VCM/nHAC/CSH disc in the center of agar matrix for 16 hours of incubation. On HE and Masson histological analysis at 12 weeks after implantation, single islands of new trabecular bone formed, and a number of active nonaligned osteocytes and osteoblasts at the periphery of bone trabecula were frequently observed in the VCM/nHAC/CSH group. No signs of necrosis and inflammation were found in the group of $\mathrm{VCM} / \mathrm{nHAC} / \mathrm{CSH}$ at this time. By contrast, all rabbits revealed evidence of chronic infections, and there were many bone necroses in the control group. Histological observation in this study demonstrated the effectiveness of treatment in an experimental 


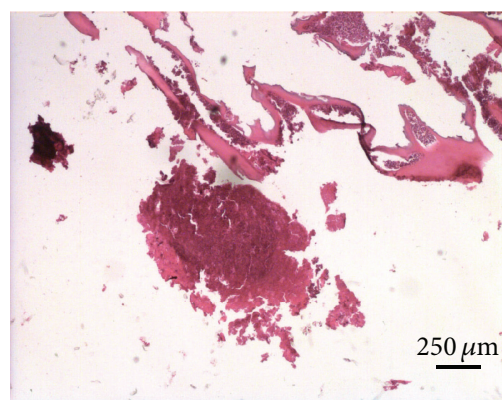

(a)

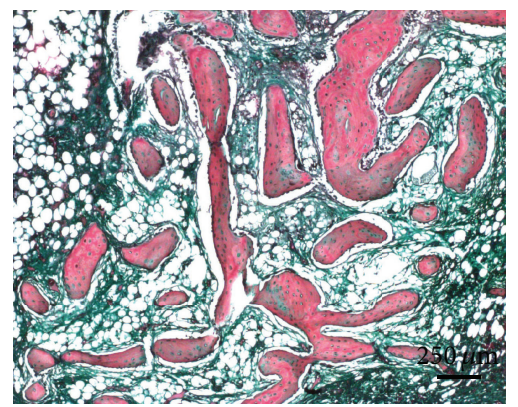

(d)

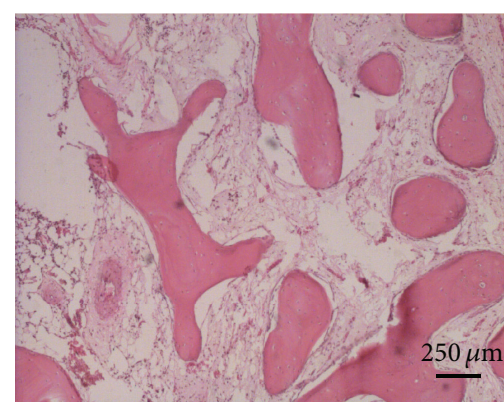

(b)

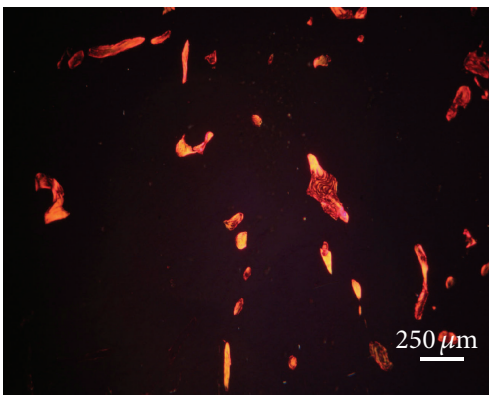

(e)

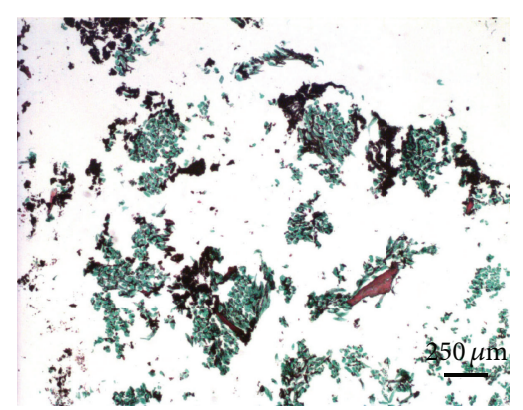

(c)

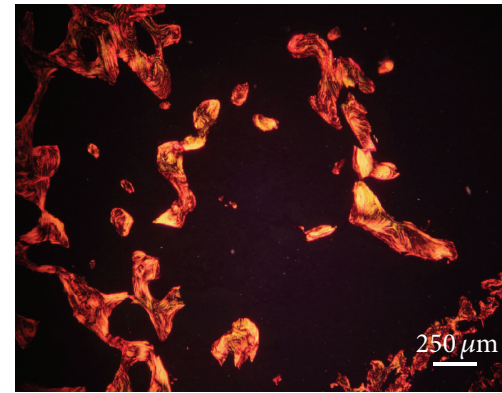

(f)

Figure 3: Histological cross sections $(4 \mu \mathrm{m})$ after infection with MRSA (40x): (a) cross section (HE) and (c) cross section (Masson) of nHAC/CSH group after 12 weeks of implantation; (b) cross section (HE) and (d) cross section (Masson) of VCM/nHAC/CSH group after 12 weeks of treatment and picrosirius red stained cross section after 12 weeks of implantation; (e) nHAC/CSH group; (f) VCM/nHAC/CSH group.

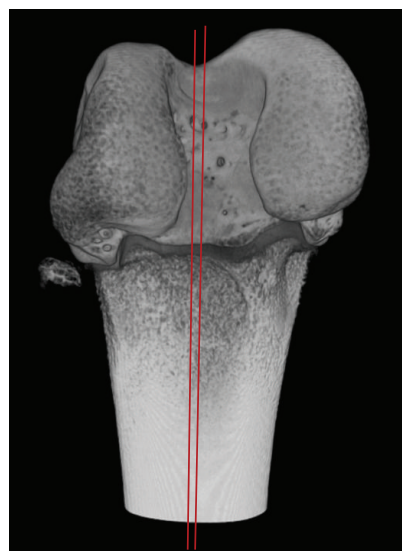

(a)

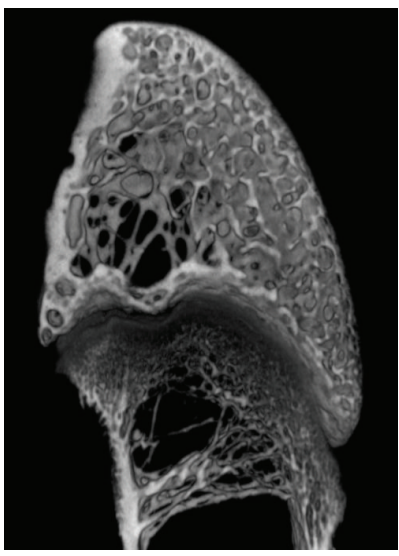

(b)

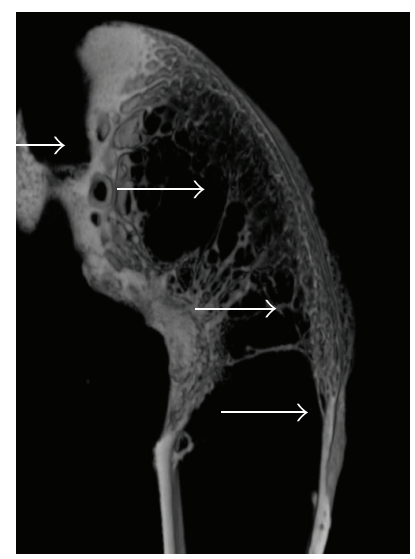

(c)

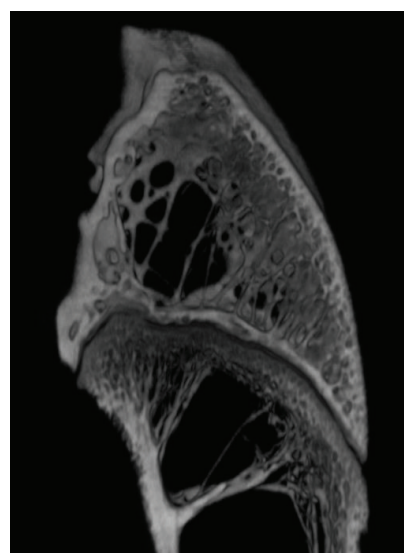

(d)

FIGURE 4: Micro-CT graphs taken 12 weeks after focal debridement. (a) Cross section position (red line), (b) normal bone, (c) nHAC/CSH group, and (d) VCM/nHAC/CSH group.

model of chronic bone infection caused by MRSA within 12 weeks.

Picrosirius red staining is one of the best techniques of collagen histochemistry. Sirius red enhances the birefringence in oriented collagens as it attaches to collagens in parallel; then we can see the red-orange colored light from sirius red stained collagens in polarized light microscopy. As the enhancement of birefringence is limited in fibrous tissues, this method can be used to identify fibrous collagen. In this study, the tissues were fixed in a solution of $10 \%$ neutral buffered formalin, embedded in paraffin, sectioned at a thickness of $5 \mu \mathrm{m}$, and stained with picrosirius red. There were more fibrous tissues in the VCM/nHAC/CSH group than that in nHAC/CSH group in picrosirius red stained cross section after infection with MRSA following 12 weeks of treatment as shown in Figures 3(e) and 3(f).

The results also were confirmed by the micro-computed tomography graphs of implanted materials on the antibacterial bone defect as shown in Figure 4. After 12 weeks, the bone tissues were destroyed in different places of rabbit 
femoris (pointed with the arrows in Figure 4(c)) because of infection without drug release in $\mathrm{nHAC/CSH}$ group. But, in the treatment group (Figure $4(\mathrm{~d})$ ), the bone reconstruction was better than control group (Figure $4(\mathrm{c})$ ) and close to normal femoris bone (Figure 4(b)).

All results above suggested that the inflammation may actually inhibit the growth of bone tissue in nHAC/CSH group, and the treatment of inflammation along with bone repair was effective in VCM/nHAC/CSH group.

The best treatment of contaminated or infected bone defects, such as chronic osteomyelitis, is to control infection and repair bone defect at the same time. This requires an osteoinductive bone graft composite with ideal release antibiotic capabilities, mechanical properties, and other related properties. In this study, the $\mathrm{nHAC/CSH}$ was used as a carrier of vancomycin (VCM) for the treatment of osteomyelitis, and the VCM/nHAC/CSH composite has ideal self-setting, antibacterial, porous, degradable, good mechanical properties, and better osteogenic activity. So the materials can act not only as void filler facilitating tissue regeneration but also as carrier for inhibiting infection in the healing process.

The bioactivity of the scaffold was determined by the components such as $\mathrm{CSH}$ and nHAC, of which $\mathrm{CSH}$ as the main ingredient has been used in bone augmentation for many years in virtue of its self-setting ability in situ as well as filling the defect. One major drawback in the use of calcium sulphate is insufficient osteogenic activity. Calcium ions were released during dissolution of calcium sulfate. Its dissolution leads to acidic microenvironment responsible for local inflammatory processes at the site of implantation in human bone. Inflammatory tissue was found to disappear after 60 days in bone but to remain in soft tissue implantation sites of white New Zealand rabbits [33]. On the other hand, local increases in calcium ion concentration may affect osteoblast genesis and function, and they may act as a stimulus to osteoblast differentiation. Besides, in live tissue, HA and collagen are the major mineral and organic component of human bones. Recently, various hydroxyapatite (HA) or collagen based composites were developed as potential biomaterials for bone substitutes due to their compositional analogy to bone [34]. Some research uses modified HA as antimicrobial coatings or carrier [35]. In this paper, nHAC was added to calcium sulphate to enhance biocompatibility of calcium sulphate in a composite material, which was developed based on biomimetically synthetic mineralized collagen fibrils. The inorganic phase in the composite is carbonate-substituted HA with low crystallinity as well as nanometer size, and organic phase of type I collagen matrix has a characteristic quarter staggered arrangement of tropocollagen molecules assembled and aligned with an axial period of approximately $67 \mathrm{~nm}$. The matrix serves as a template for orderly deposition of mineral platelets [28]. Studies have demonstrated that nanostructured materials with cell favorable surface properties may promote greater amounts of specific protein interactions to more efficiently stimulate new bone growth compared to conventional materials [29]. The composite has been proven to be with unique osteoinduction and osteoconduction and successfully applied in clinical treatment.
Since the 1980s, vancomycin has become the first choice for treating refractory hip infections because of its high efficacy against gentamicin-resistant bacteria, its rare bacterial resistance, and its low incidence of side effect [8]. In this study, vancomycin can be mixed with powdered $n \mathrm{HAC} / \mathrm{CSH}$ at the site of bone defect and sustained release on the course of operation. However, loading is not limited to one specific antibiotic (e.g., tobramycin or gentamicin) but can be done according to antibiograms offering individual treatment options. Calcium sulphate's capillary porosity also can be used for impregnation of already hardened calcium sulphate pellets with various antibiotic solutions, which would be reported in our subsequent research.

In in vivo study, after three weeks of induction of infection and 12 weeks of treatment with VCM/nHAC/CSH, no evidence of infection or foreign body reaction was observed. The scaffold had almost been completely degraded and a lot of new trabecular bones had formed in the implant site. By contrast, all rabbits in the control groups of $n \mathrm{HAC} / \mathrm{CSH}$ showed evidence of chronic infections.

\section{Conclusion}

In this study, we first used the VCM/nHAC/CSH bone substitute as a degradable local antibiotic delivery system for the treatment of chronic osteomyelitis. The implants were successful as vancomycin carriers in inhibiting infection in rabbits osteomyelitis mode during the course of this study. Moreover, the implants performed excellent biocompatibility. Our results suggested that the implants can be considered a new system for local vancomycin delivery and the effectiveness of $\mathrm{VCM} / \mathrm{nHAC} / \mathrm{CSH}$ bone substitute in the treatment of $S$. aureus induced chronic osteomyelitis and simultaneously stimulated bone regeneration.

\section{Conflict of Interests}

The authors declare that there is no conflict of interests regarding the publication of this paper.

\section{Acknowledgments}

This work is in part supported by the Natural Science Foundation for Young Scientists of Shanxi Province (no. 20140210396), the Qualified Personnel Foundation of Taiyuan University of Technology (QPFT) (no. tyut-rc201270a), the Youth Foundation of Taiyuan University of Technology (nos. 120504020102, 2013Z020, and 2014TD066), the National HighTech Program (no. 2011AA030105), the Technical Services Project of Taiyuan University of Technology (no. 143230043J), the National Science Foundation of China (nos. 50830102, 51303119, 21161003, and 20701010), MOST of China (no. 2011DFA31430), Grants of Science and Technology of Guangdong Province (no. 2010B031500005), and the Natural Science Foundation of Jiangsu Province (BK20130309). 


\section{References}

[1] X. M. Li, C. A. van Blitterswijk, Q. L. Feng, F. Z. Cui, and F. Watari, "The effect of calcium phosphate microstructure on bone-related cells in vitro," Biomaterials, vol. 29, no. 23, pp. 3306-3316, 2008.

[2] X. M. Li, H. F. Liu, X. F. Niu et al., "Osteogenic differentiation of human adipose-derived stem cells induced by osteoinductive calcium phosphate ceramics," Journal of Biomedical Materials Research Part B: Applied Biomaterials, vol. 97, no. 1, pp. 10-19, 2011.

[3] X. M. Li, Y. Huang, L. S. Zheng et al., "Effect of substrate stiffness on the functions of rat bone marrow and adipose tissue derived mesenchymal stem cells in vitro," Journal of Biomedical Materials Research Part A, vol. 102, no. 4, pp. 1092-1101, 2014.

[4] C. L. Nelson, S. G. Hickmon, and B. H. Harrison, "Elution characteristics of gentamicin-PMMA beads after implantation in humans," Orthopedics, vol. 17, no. 5, pp. 415-416, 1994.

[5] S. Deb, R. Doiron, L. DiSilvio, S. Punyani, and H. Singh, "PMMA bone cement containing a quaternary amine comonomer with potential antibacterial properties," Journal of Biomedical Materials Research B: Applied Biomaterials, vol. 85, no. 1, pp. 130-139, 2008.

[6] D. Neut, O. S. Kluin, J. Thompson, H. C. van der Mei, and H. J. Busscher, "Gentamicin release from commercially-available gentamicin-loaded PMMA bone cements in a prosthesisrelated interfacial gap model and their antibacterial efficacy," BMC Musculoskeletal Disorders, vol. 11, article 258, 2010.

[7] H. L. Tan, W. T. Lin, and T. T. Tang, "The use of antimicrobialimpregnated PMMA to manage periprosthetic infections: controversial issues and the latest developments," International Journal of Artificial Organs, vol. 35, no. 10, pp. 832-839, 2012.

[8] S. G. Yan, X. Z. Cai, W. Q. Yan, X. S. Dai, and H. B. Wu, "Continuous wave ultrasound enhances vancomycin release and antimicrobial efficacy of antibiotic-loaded acrylic bone cement in vitro and in vivo," Journal of Biomedical Materials Research B: Applied Biomaterials, vol. 82, no. 1, pp. 57-64, 2007.

[9] Z. P. Xie, X. Liu, W. T. Jia, C. Q. Zhang, W. H. Huang, and J. Q. Wang, "Treatment of osteomyelitis and repair of bone defect by degradable bioactive borate glass releasing vancomycin," Journal of Controlled Release, vol. 139, no. 2, pp. 118-126, 2009.

[10] M. Larsson, A. Bergstrand, L. Mesiah, C. Van Vooren, and A. Larsson, "Nanocomposites of polyacrylic acid nanogels and biodegradable polyhydroxybutyrate for bone regeneration and drug delivery," Journal of Nanomaterials, vol. 2014, Article ID 371307, 9 pages, 2014.

[11] X. Lian, H. Liu, X. Wang, S. Xu, F. Cui, and X. Bai, "Antibacterial and biocompatible properties of vancomycin-loaded nanohydroxyapatite/collagen/poly (lactic acid) bone substitute," Progress in Natural Science: Materials International, vol. 23, no. 6, pp. 549-556, 2013.

[12] M.-P. Ginebra, C. Canal, M. Espanol, D. Pastorino, and E. B. Montufar, "Calcium phosphate cements as drug delivery materials," Advanced Drug Delivery Reviews, vol. 64, no. 12, pp. 1090-1110, 2012.

[13] U. Joosten, A. Joist, G. Gosheger, U. Liljenqvist, B. Brandt, and C. Von Eiff, "Effectiveness of hydroxyapatite-vancomycin bone cement in the treatment of Staphylococcus aureus induced chronic osteomyelitis," Biomaterials, vol. 26, no. 25, pp. 52515258, 2005.

[14] H. A. Doty, M. R. Leedy, H. S. Courtney, W. O. Haggard, and J. D. Bumgardner, "Composite chitosan and calcium sulfate scaffold for dual delivery of vancomycin and recombinant human bone morphogenetic protein-2," Journal of Materials Science: Materials in Medicine, vol. 25, no. 6, pp. 1449-1459, 2014.

[15] M. V. Thomas and D. A. Puleo, "Calcium sulfate: properties and clinical applications," Journal of Biomedical Materials Research Part B: Applied Biomaterials, vol. 88, no. 2, pp. 597-610, 2009.

[16] D. Stubbs, M. Deakin, P. Chapman-Sheath et al., "In vivo evaluation of resorbable bone graft substitutes in a rabbit tibial defect model," Biomaterials, vol. 25, no. 20, pp. 5037-5044, 2004.

[17] G. Orsini, J. Ricci, A. Scarano et al., "Bone-defect healing with calcium-sulfate particles and cement: an experimental study in rabbit," Journal of Biomedical Materials Research Part B: Applied Biomaterials, vol. 68, no. 2, pp. 199-208, 2004.

[18] Z. G. Huan and J. Chang, "Self-setting properties and in vitro bioactivity of calcium sulfate hemihydrate-tricalcium silicate composite bone cements," Acta Biomaterialia, vol. 3, no. 6, pp. 952-960, 2007.

[19] P. Wang, E.-J. Lee, C.-S. Park et al., "Calcium sulfate hemihydrate powders with a controlled morphology for use as bone cement," Journal of the American Ceramic Society, vol. 91, no. 6, pp. 2039-2042, 2008.

[20] C. Du, F. Z. Cui, X. D. Zhu, and K. De Groot, “Three-dimensional nano-HAp/collagen matrix loading with osteogenic cells in organ culture," Journal of Biomedical Materials Research, vol. 44, no. 4, pp. 407-415, 1999.

[21] F.-Z. Cui, Y. Li, and J. Ge, "Self-assembly of mineralized collagen composites," Materials Science and Engineering R: Reports, vol. 57, no. 1-6, pp. 1-27, 2007.

[22] C. Du, F. Z. Cui, W. Zhang, Q. L. Feng, X. D. Zhu, and K. de Groot, "Formation of calcium phosphate/collagen composites through mineralization of collagen matrix," Journal of Biomedical Materials Research, vol. 50, no. 4, pp. 518-527, 2000.

[23] S. S. Liao, F. Z. Cui, W. Zhang, and Q. L. Feng, "Hierarchically biomimetic bone scaffold materials: nano-HA/collagen/PLA composite," Journal of Biomedical Materials Research Part B: Applied Biomaterials, vol. 69, no. 2, pp. 158-165, 2004.

[24] S. S. Liao and F.-Z. Cui, "In vitro and in vivo degradation of mineralized collagen-based composite scaffold: nanohydroxyapatite/collagen/poly(L-lactide)," Tissue Engineering, vol. 10, no. 1-2, pp. 73-80, 2004.

[25] Z. Chen, H. Liu, X. Liu, and F.-Z. Cui, "Injectable calcium sulfate/mineralized collagen-based bone repair materials with regulable self-setting properties," Journal of Biomedical Materials Research Part A, vol. 99, no. 4, pp. 554-563, 2011.

[26] X. M. Li, H. F. Liu, X. F. Niu et al., “The use of carbon nanotubes to induce osteogenic differentiation of human adipose-derived MSCs in vitro and ectopic bone formation in vivo," Biomaterials, vol. 33, no. 19, pp. 4818-4827, 2012.

[27] X. Li, H. Gao, M. Uo et al., "Maturation of osteoblast-like SaoS2 induced by carbon nanotubes," Biomedical Materials, vol. 4, no. 1, Article ID 015005, 2009.

[28] C. Du, F. Z. Cui, Q. L. Feng, X. D. Zhu, and K. De Groot, “Tissue response to nano-hydroxyapatite/collagen composite implants in marrow cavity," Journal of Biomedical Materials Research, vol. 42, no. 4, pp. 540-548, 1998.

[29] X. M. Li, L. Wang, Y. B. Fan, Q. L. Feng, F.-Z. Cui, and F. Watari, "Nanostructured scaffolds for bone tissue engineering," Journal of Biomedical Materials Research Part A, vol. 101, no. 8, pp. 24242435, 2013. 
[30] Z. Chen, H. Liu, X. Liu et al., "Improved workability of injectable calcium sulfate bone cement by regulation of selfsetting properties," Materials Science and Engineering C, vol. 33, no. 3, pp. 1048-1053, 2013.

[31] X. Lian, X. Wang, and F. Cui, "In vitro antibacterial properties of vancomycin-loaded nano-hydroxyapatite/collagen/ calcium sulfate hemihydrates (VCM/nHAC/CSH) bone substitute," Materials Science Forum: Advances in Functional and Electronic Materials, vol. 745-746, pp. 6-12, 2013.

[32] W. Zhang, S. S. Liao, and F. Z. Cui, "Hierarchical self-assembly of nano-fibrils in mineralized collagen," Chemistry of Materials, vol. 15, no. 16, pp. 3221-3226, 2003.

[33] M. A. Rauschmann, T. A. Wichelhaus, V. Stirnal et al., "Nanocrystalline hydroxyapatite and calcium sulphate as biodegradable composite carrier material for local delivery of antibiotics in bone infections," Biomaterials, vol. 26, no. 15, pp. 2677-2684, 2005.

[34] X. M. Li, Q. L. Feng, X. H. Liu, W. Dong, and F. Z. Cui, "Collagen-based implants reinforced by chitin fibres in a goat shank bone defect model," Biomaterials, vol. 27, no. 9, pp. 19171923, 2006.

[35] C. S. Ciobanu, C. L. Popa, and D. Predoi, "Sm:HAp nanopowders present antibacterial activity against Enterococcus faecalis," Journal of Nanomaterials, vol. 2014, Article ID 780686, 9 pages, 2014. 

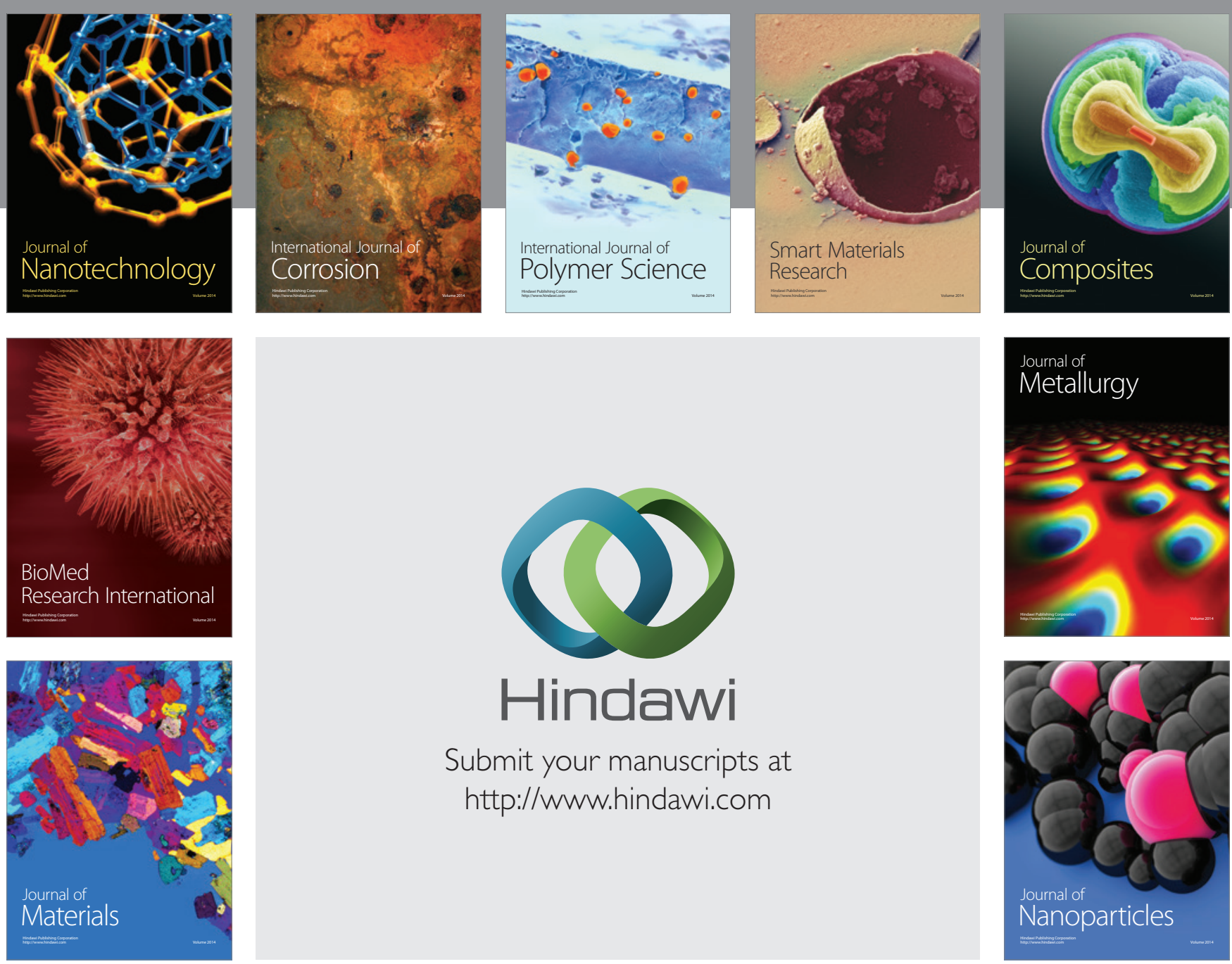

Submit your manuscripts at http://www.hindawi.com
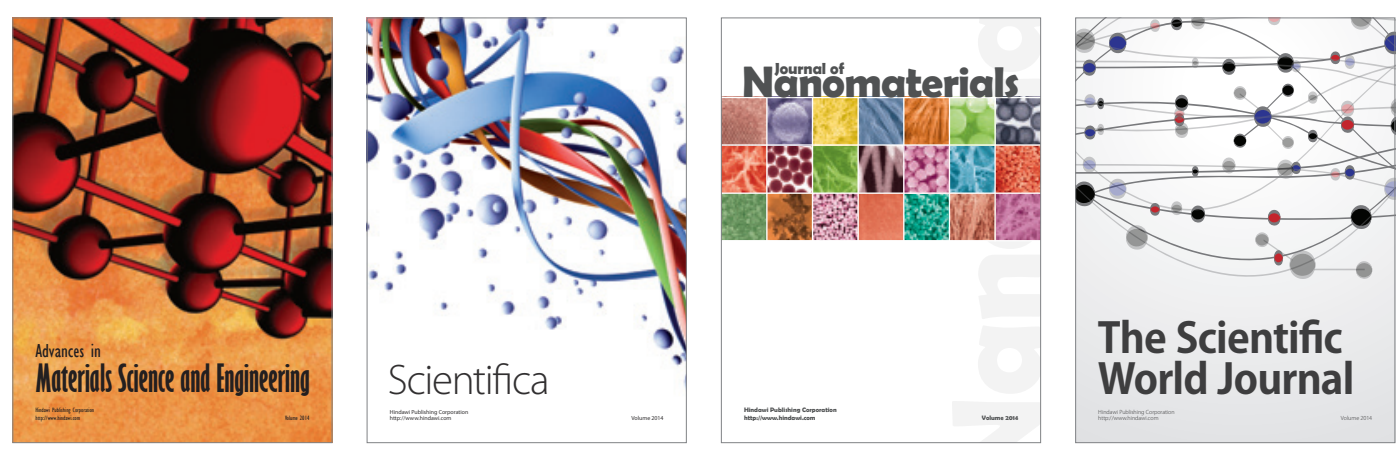

\section{The Scientific World Journal}
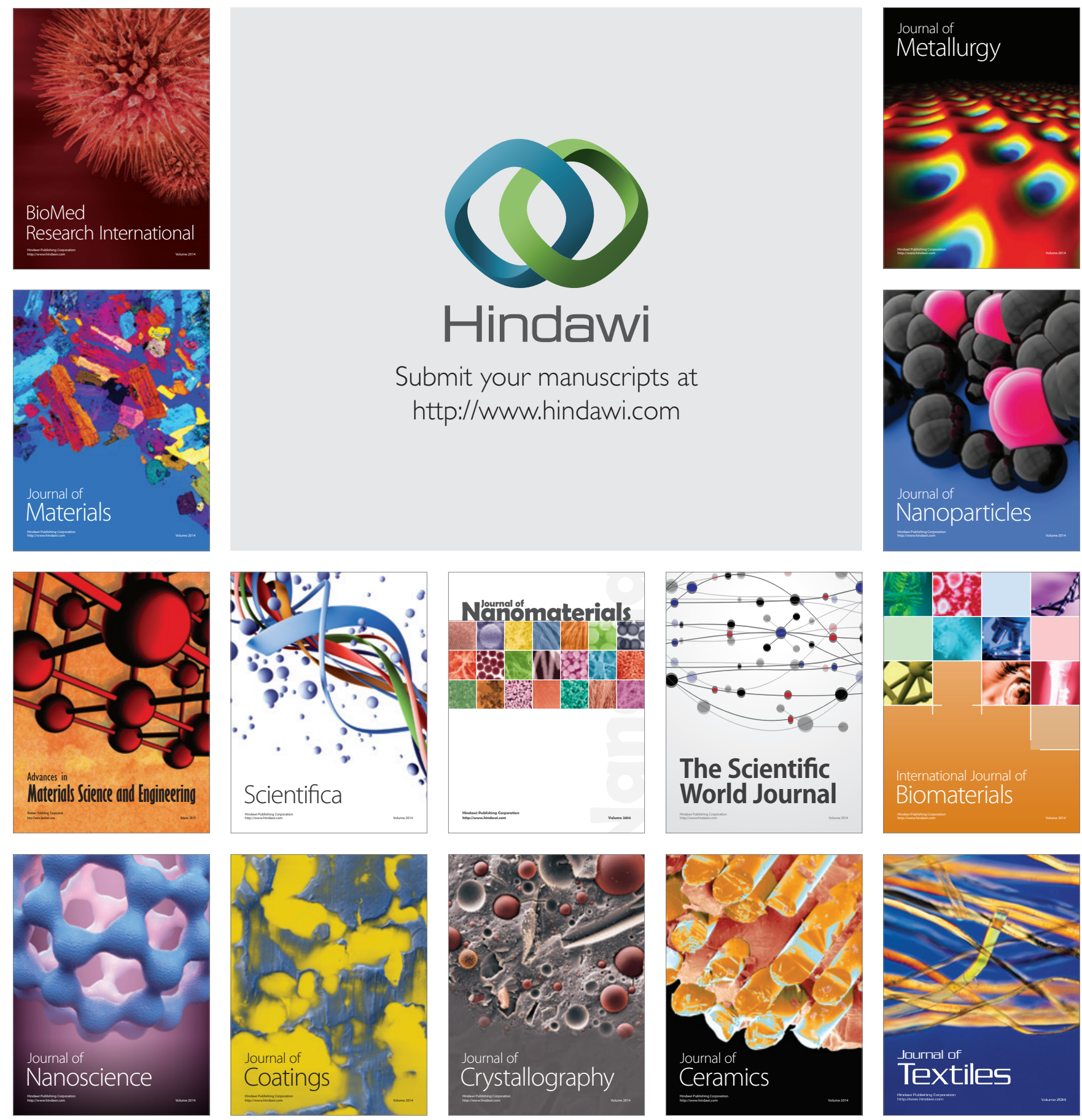